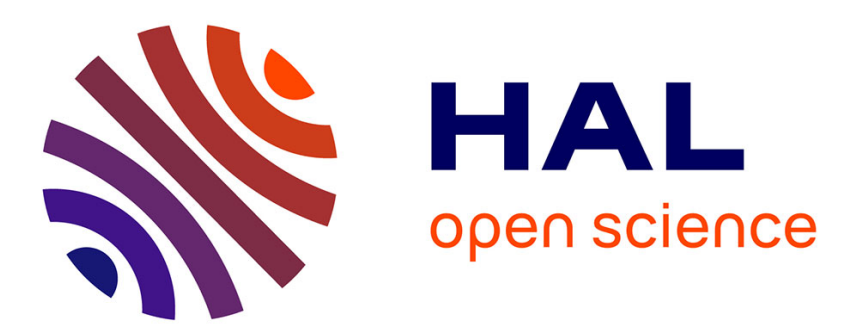

\title{
A Miniature Biomimetic Robotic Fish and Its Realtime Path Planning
}

Chao Zhou, Zhiqiang Cao, Shuo Wang, Xiang Dong, Min Tan

\section{To cite this version:}

Chao Zhou, Zhiqiang Cao, Shuo Wang, Xiang Dong, Min Tan. A Miniature Biomimetic Robotic Fish and Its Realtime Path Planning. 6th International Conference on Field and Service Robotics - FSR 2007, Jul 2007, Chamonix, France. inria-00194853

\section{HAL Id: inria-00194853 \\ https://hal.inria.fr/inria-00194853}

Submitted on 7 Dec 2007

HAL is a multi-disciplinary open access archive for the deposit and dissemination of scientific research documents, whether they are published or not. The documents may come from teaching and research institutions in France or abroad, or from public or private research centers.
L'archive ouverte pluridisciplinaire HAL, est destinée au dépôt et à la diffusion de documents scientifiques de niveau recherche, publiés ou non, émanant des établissements d'enseignement et de recherche français ou étrangers, des laboratoires publics ou privés. 


\title{
A Miniature Biomimetic Robotic Fish and Its Realtime Path Planning ${ }^{\star}$
}

\author{
Chao Zhou, Zhiqiang Cao, Shuo Wang, Xiang Dong, and Min Tan \\ Laboratory of Complex Systems and Intelligence Science, Institute of \\ Automation,Chinese Academy of Sciences, Beijing, China \\ zhouchao@compsys.ia.ac.cn
}

Summary. This paper proposes a novel miniature biomimetic robotic fish based on single link with compact structure, high maneuverability and multiple sensors. The robotic fish mimics the motion of Thunniform mode, and the methods of propelling and turning are given. The information provided by infrared sensors and photosensitive sensors are analyzed and applied to the algorithms of obstacle avoidance, the light source searching and the dynamic light source tracking. Experimental results are given to show the motion ability and the validity of the proposed algorithm.

\section{Introduction}

There are more and more productive underwater activities and research work, and the Autonomous Underwater Vehicle (AUV) is paid more attentions on. Fish exhibit the advantages of the high efficiency, high maneuverability and low noise, which has the advantages over conventional marine vehicles powered by rotary propellers with the same power consumption. Roboticists explore robotic fish to develop small-size, efficient, maneuverable, and low noise AUV.

Investigation on biomimetic robotic fish systems has provided significant insights into both theory and application of robotics in recent years, whose appealing nature involves higher efficiency, more remarkable maneuverability and quieter actuation[1][2]. These advantages are of great benefit to applications in marine and military fields. Many theories are proposed to explore secrets of fish swimming mechanisms and summarize driving modes of fish motions [3]-[5]. Based on these theories, many prototypes of biomimetic robotic fish have been developed [1] [6]-[9].

Considering that there are some special occasions under the water, such as pipelines, gaps, the development of miniature robotic fish with high maneuverability and adaptability is of great advantages. On the other hand, the

\footnotetext{
* This work is funded by the National Natural Science Foundation of China (No. 60635010, No.60605026, No.50475179), 863 Project (No.2006AA11Z225), and CASIA Innovation Fund for Young Scientists.
} 
miniature robotic fish is cheap enough to be mass-produced, which may enhance the quality of the solution by cooperation among them. The miniature robotic fish should be elaborately designed because all the mechanical structures, control units, sensors, communication module and power supply are integrated into a very limited space, which brings new challenge.

There are some miniature robotic fish prototypes. Some of them utilize new materials as the actuators, such as ICPF, which can reduce the volume largely. However, the power dissipation is so much that the power source should be outside [7] [10], which will restrict the robotic fish's motion greatly because of the power line. Some miniature swimming robots are designed for special purposes, and the motion ability is suitable for special environment, for example, for the medical purposes [11], but it may not adapt the general task.

In this paper, the design of a miniature biomimetic robotic fish is given based on single link undulating, which is only 15 centimeters long. The method of propelling and turning are proposed based on the motion law of Thunniform mode. The robotic fish may execute tasks independently and autonomously. The algorithms of obstacle avoidance and light source tracking based on the infrared sensors and photosensitive sensors are introduced.

\section{The design of the robotic fish and motion control}

\subsection{The structure of the robotic fish}

According to the wavelength and the amplitude envelope of the propulsive wave underlying fish's behavior, the fish is divided into five different modes: Anguilliform mode, Subcarangiform mode, Carangiform mode, Thunniform mode, Ostraciiform mode [12].

Thunniform mode is by far the most efficient locomotion mode evolved in the aquatic environment, where thrust is generated with a lift-based method, allowing high cruising speeds to be maintained for long periods. Significant lateral movements occur only at the caudal fin (producing more than $90 \%$ of the thrust) and at the area near the narrow peduncle. In addition, the reduction of the length of the undulating part will simplify the structure, and reduce the volume on the whole. A novel Thunniform mode-based miniature robotic fish is designed, as shown in Fig.1. A servo motor is adopted to drive a lunate caudal fin as the propeller of the robotic fish, according to fish outline and its motion characteristics. The servo is agile and controllable in movement without additional driver, and more energy is saved than that of the driving unit adopting some new materials such as SMA.

Because of the volume restriction, all assembly units are highly costeffective ones. The forebody is fish-shape thin hard plastic and a servo is fixed on it. The controller integrates the functions of information acquisition and processing, communication, control and motion decision to improve the 


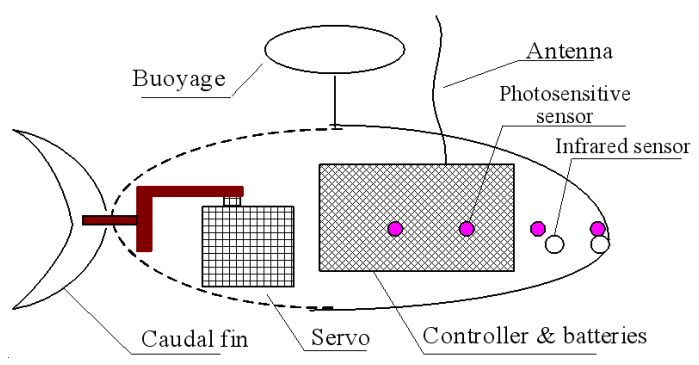

Fig. 1. The schematic structure of the robotic fish

integration level and reduce the volume and power dissipation. The tail is cover by thin flexible rubber film as waterproofer, which has little effect on the undulating. The wireless signal is sent by an antenna out of the water. The Li-Polymer Batteries is chosen for the high energy density. In the system of sensors, the infrared switch sensors are selected instead of the sonar sensors because of the volume. The average density of the robotic fish except the buoyage is made a little higher than water by fixed weight on bottom, and the robotic fish will float on water with the buoyage so that all the body is under water, which is favourable to the propelling and stability.

\subsection{The structure of the robotic fish}

Thunniform mode motion involves the undulation of the entire body, whose large amplitude undulation is mainly confined to the last $1 / 3$ part of the body. A Thunniform propulsive model is given to describe the motion in the frame of polar coordinate for caudal motion as shown in Fig.2.

$$
A_{\text {body }}(t)=A m p \cdot \sin (\omega t)
$$

where the $A_{\text {body }}$ is the realtime angle of the tail, $A m p$ is the undulation amplitude, $\omega$ is the frequency of the wave $(\omega=2 \pi f=2 \pi / T)$.

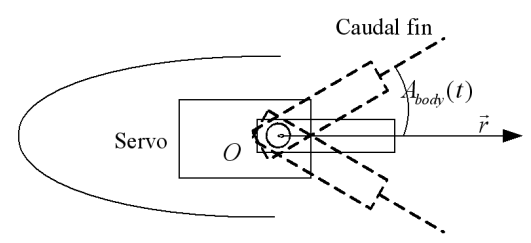

Fig. 2. The polar coordinate for caudal motion

(1) provides a method to design the robotic fish: choose the parameters including $A m p, \omega$, to determine the proper body wave and then develop a 
mechanical structure to fit the curve so that the fish's locomotion may be emulated. Discrete (1), then we have:

$$
A_{\text {body }}(i)=A m p \cdot \sin \left(\frac{2 \pi}{M} i\right)
$$

where $i$ is the variable of spline curve sequence, $M$ is the body-wave resolution that represents the discrete degree of the overall traveling wave.

The interval of time and the movement distance between every two points is restricted by the maximum angle velocity and amplitude of the servo, which is described as follows.

$$
\left\{\begin{array}{l}
\max \left\{\left|A_{\text {body }}(i)-A_{\text {body }}(i-1)\right|\right\} \cdot f \cdot M<\Omega_{\max } \\
A_{\text {body }}(i) \leq A m p_{\max }
\end{array} \quad 1 \leq i \leq M\right.
$$

where $\Omega_{\max }, A m p_{\max }$ are the maximum angular velocity and amplitude respectively. $\max \left\{A_{\text {body }}(i)\right\} \leq A m p$, so $A m p$ is proportional to $\max \left\{\mid A_{\text {body }}(i)-\right.$ $\left.A_{\text {body }}(i-1) \mid\right\}$ with $M$ being constant. Let:

$$
A m p=c \cdot \max \left\{\left|A_{\text {body }}(i)-A_{\text {body }}(i-1)\right|\right\}
$$

Then the constraint equations between the Amp and $f$ are obtained:

$$
\left\{\begin{array}{l}
A m p \leq A m p_{\max } \\
f \leq c \omega_{\max } /(M \cdot A m p)
\end{array}\right.
$$

The turn of the fish, according to the observation, is implemented by changing the axis of the body undulation. Based on it, the direction of the robotic fish may be controlled. A certain angle is superposed on the caudal fin. It is necessary to insure that the undulating rule marches (2) when turning, Rewriting (2), we have:

$$
A_{\text {body }}(i)=A m p \cdot \sin \left(\frac{2 \pi}{M} i\right)+A_{t u r n}
$$

where $A_{\text {turn }}$ is the deflection angle of the caudal fin. This turning method is used in continuing, stable turning in the movement. The motion of servo should also fit the constraint of (3)

\section{The realtime path planning based on sensors information}

The path planning is necessary for the robotic fish to complete the task. Considering that the limitation of the volume, infrared sensors are chosen to detect the obstacle, and the photosensitive sensors to detect the light source. Firstly, the inertial frame of reference for the motion is defined as shown in Fig.3. $O$ is the center of inertia of the robotic fish, and is the direction of the rigid forebody. 


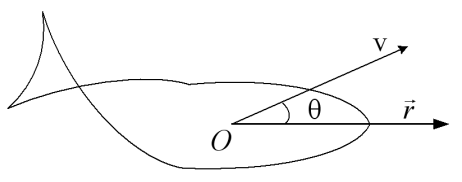

Fig. 3. The inertial frame of reference

The motion state of the robotic fish $S_{j}$ at $t=j$ includes the velocity and direction, which is shown in (7).

$$
S_{j}=\left[v_{j}, \theta_{j}\right]^{T}
$$

We denote with $\mathrm{F}$ the motion function and we have:

$$
S_{j}=F\left(S_{j-1}, \cdots, S_{j-n}, A c t_{j}\right)
$$

where $A c t_{j}$ is the action of the robotic fish at $t=j$,

$$
A c t_{j}=\left[A m p, f, A_{\text {turn }}\right]_{j}^{T}
$$

It means that the action of robotic fish includes the frequency, amplitude, and deflection angle. The C-turn is not involved because it is not suitable for the path planning. In the implementation, all the three parameters are discrete and numbered, and the sets of them $\operatorname{are}\left\{A m p^{n}\right\},\left\{f^{m}\right\},\left\{A_{\text {turn }}^{l}\right\}$, so the Act is numbered, therefore, all the action of the robotic fish is:

$$
\Phi_{\text {act }}=\left\{A c t \mid A m p \in\left\{A m p^{n}\right\}, f \in\left\{f^{m}\right\}, A_{\text {turn }} \in\left\{A_{\text {turn }}^{l}\right\}\right\}
$$

\subsection{The algorithm of obstacle avoidance}

Three infrared sensors are installed on the robotic fish, the output of them are Boolean variables and labeled as $I r_{l}$ (left), $I r_{m}$ (middle), $I r_{r}$ (right). '1'means there is obstacle. The distribution of the sensors is shown in Fig.4. $\theta$ is the angle between the velocity $v$ and $\mathbf{r} . \alpha_{r}, \alpha_{m}$ and $\alpha_{l}$ are the detecting directions of three sensors, respectively.

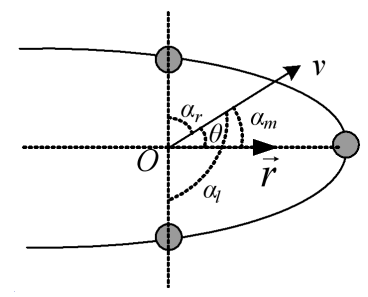

Fig. 4. The distribution of infrared sensors 
The purpose of avoiding obstacle algorithm is to select the safest direction for robotic fish. The risk factor function at $t=j$ is defined as follows:

$$
\begin{gathered}
\operatorname{Risk}_{j}=-\left(\operatorname{Ir}_{l, j} \cdot \alpha_{l, j}+\operatorname{Ir}_{m, j} \cdot \alpha_{m, j}+I r_{r, j} \cdot \alpha_{r, j}\right) \\
=-\left[\operatorname{Ir}_{l, j} \cdot\left(90^{\circ}+\theta_{j}\right)+I r_{m, j} \cdot\left|\theta_{j}\right|+I r_{r, j} \cdot\left(90^{\circ}-\theta_{j}\right)\right]
\end{gathered}
$$

The smaller Risk $k_{j}$ is, the smaller the collision probability of robotic fish is. So the algorithm is to calculate the robotic fish action at $t=j+1, A c t_{j+1}^{*}=$ $\left[A m p^{*}, f^{*}, d_{\text {turn }}^{*}\right]^{T}$, which satisfy:

$$
\begin{aligned}
& \min _{\text {Act }_{j+1} \in \Phi_{a c t}}\left\{\operatorname{Risk}_{j+1}\right\} \\
& =\min _{A c t_{j+1} \in \Phi_{a c t}}\left\{-\left[I r_{l, j+1} \cdot\left(90^{\circ}+\theta_{j+1}\right)+I r_{m, j+1} \cdot\left|\theta_{j+1}\right|+I r_{r, j+1} \cdot\left(90^{\circ}-\theta_{j+1}\right)\right]\right\}
\end{aligned}
$$

The information of sensors, $I r_{l, j+1}, I r_{m, j+1}$ and $I r_{r, j+1}$ are unknown at $t=j$, so the values at $t=j, I r_{l, j}, I r_{m, j}$ and $I r_{r, j}$ are accepted for estimation. Thus,

$$
A c t_{j+1}^{*}=\arg \min _{A c t_{j+1} \in \Phi_{a c t}}\left\{-\left[\operatorname{Ir}_{l, j} \cdot\left(90^{\circ}+\theta_{j+1}\right)+I r_{m, j} \cdot\left|\theta_{j+1}\right|+I r_{r, j} \cdot\left(90^{\circ}-\theta_{j+1}\right)\right]\right\}
$$

\subsection{The algorithm of light source searching}

There are seven mini photosensitive sensors installed on the rigid forebody, and the distribution of the sensors is shown in Fig.5

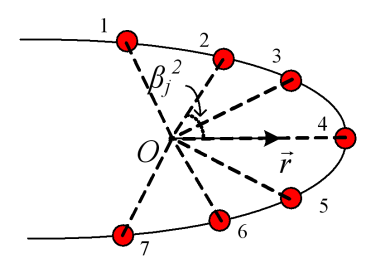

Fig. 5. The distribution of the photosensitive sensors

Label $\beta_{j}^{k}$ as the detecting directions of the $k^{t h}$ sensor at $t=j$, as $\beta_{j}^{2}$ shown in Fig.5. The light intensity detected by the $k^{t h}$ sensor is labeled as $I_{k}$ and the direction of the light source is calculated as follows:

Firstly, obtain maximum value of $I_{k}$ :

$$
I_{\max }=\max _{1 \leq k \leq 7}\left\{I_{k}\right\}
$$


If $I_{\max }<I_{e n v}$, it means that that are no light source, where $I_{e n v}$ is the light intensity of environment.

else

$$
K=\arg \max _{1 \leq k \leq 7}\left\{I_{k}\right\}
$$

Secondly, determine the direction of the light source at $t=j$ :

$$
\beta_{j}^{*}=\frac{\sum_{k=K-1}^{k=K+1}\left(I_{k}-I_{e n v}\right) \cdot \beta_{j}^{k}}{\sum_{k=K-1}^{k=K+1}\left(I_{k}-I_{e n v}\right)}
$$

From (16), it is seen that only $K^{t h},(K-1)^{t h},(K+1)^{t h}$ sensors are considered because the robotic fish body rocks about when it swims, and the detecting for the light intensity is disturbed. Define the photokinesis function:

$$
\text { Light }_{j}=\left|\theta_{j}-\beta_{j}^{*}\right|
$$

Thus, the photokinesis action for the robotic fish att $=j+1$ is:

$$
\begin{gathered}
A c t_{j+1}^{*}=\arg \min _{\forall A c t_{j+1} \in \Phi_{a c t}}\left\{\text { Light }_{j+1}\right\} \\
=\arg \underset{\forall A c t_{j+1} \in \Phi_{a c t}}{ }\left\{\left|\theta_{j+1}-\beta_{j+1}^{*}\right|\right\}
\end{gathered}
$$

As well, $\beta_{j+1}^{*}$ are not known at $=j$, and $\beta_{j}^{*}$ is introduced as the estimation. We have:

$$
A c t_{j+1}^{*}=\arg \min _{\forall A c t_{j+1} \in \Phi_{a c t}}\left\{\left|\theta_{j+1}-\beta_{j}^{*}\right|\right\}
$$

\subsection{The algorithm of dynamic light source tracking}

In actual task, the goal (light source) is usually dynamic, which means the robotic fish has to track light source while avoiding obstacles. When the robotic fish is close to the light source, which is both the goal and obstacle, the corresponding conflict should be considered. Because of the limited information, the robotic fish can not distinguish the obstacle and moving light source. Therefore, a multiple objectives optimization approach with self adaptive weight is proposed to solve this problem. The action of the robotic fish is decided by both the infrared sensors and photosensitive sensors:

$$
\begin{aligned}
& A c t_{j+1}^{*}=\arg \min _{\forall A c t j+1 \in \Phi_{a c t}}\left\{M_{1} \cdot \text { Light }_{j+1}+M_{2} \cdot \text { Risk }_{j+1}\right\} \\
& \quad=\arg \min _{\forall A c t_{j+1} \in \Phi_{a c t}}\left\{M_{1} \cdot\left|\theta_{j+1}-\beta_{j+1}^{*}\right|\right. \\
& \left.\quad-M_{1} \cdot\left[\operatorname{Ir}_{l, j+1} \cdot\left(90^{\circ}+\theta_{j+1}\right)+I r_{m, j+1} \cdot\left|\theta_{j+1}\right|+I r_{r, j+1} \cdot\left(90^{\circ}-\theta_{j+1}\right)\right]\right\}
\end{aligned}
$$


where $M_{1}$ and $M_{2}$ are the weights of the photosensitive sensors and infrared sensors, respectively. As well, $\beta_{j+1}^{*}$ and $I r_{l, j+1}$ are replaced by the estimated values, and we have:

$$
\begin{aligned}
& A c t_{j+1}^{*}=\arg \min _{\forall A c t_{j+1} \in \Phi_{a c t}}\left\{M_{1} \cdot\left|\theta_{j+1}-\beta_{j}^{*}\right|\right. \\
& \left.\quad-M_{1} \cdot\left[I r_{l, j} \cdot\left(90^{\circ}+\theta_{j+1}\right)+I r_{m, j} \cdot\left|\theta_{j+1}\right|+I r_{r, j} \cdot\left(90^{\circ}-\theta_{j+1}\right)\right]\right\}
\end{aligned}
$$

$M_{1}$ and $M_{2}$ are decided based on actual application. In the light tracking, it can be self adapted according to light intensity. When the light is weak, the robotic fish is far from the light source. If obstacle is detected, the algorithm of obstacle avoidance should be validated and $M_{1}>M_{2}$ in this case. When the light intensity is high, the obstacle detected may be the light source, thus $M_{1}<M_{2}$. When the light is very high, it means that the robot is very close to the goal, and the obstacle avoiding should be validated to avoid the collision with light source, therefore we have $M_{1}>M_{2}$. In the experiment, we choose a piecewise function:

$$
\begin{aligned}
& M_{1}= \begin{cases}2 & I_{\max }<I_{a} \\
0.5 & I_{a}<I_{\max }<I_{b} \\
2 & I_{\max }>I_{b}\end{cases} \\
& M_{2}=1
\end{aligned}
$$

where $I_{a}$ and $I_{b}$ are the thresholds of light intensity, which are decided by the light source.

\section{Experiments}

The developed robotic fish prototype is shown in Fig.6.

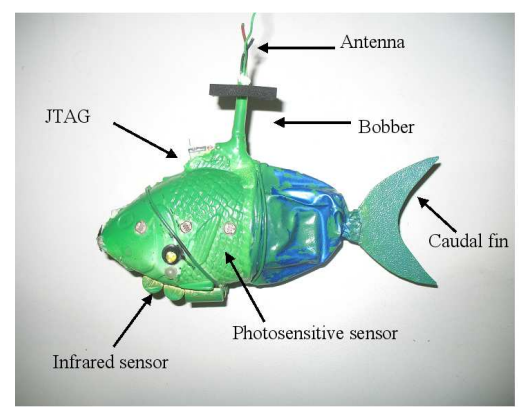

Fig. 6. The prototype of the robotic fish

The experiments are conducted in an experiment pool of $1.80 \mathrm{~m} * 1 \mathrm{~m}$ to testify the miniature biomimetic robotic fish. An overhead camera is introduced 
to capture the video of the robotic fish motion and the video information is sent to the computer to recognize and record the realtime position of the robotic fish by the help of a platform for the motion control of robotic fish, MRFS (Multiple Robot fishes coordinate System). The top right part shows the result of the recognition. The position data is filtered, and the movement track is got by Least-squares spline approximation. The relative parameters are then calculated.

Experiment 1: Testing the velocity

According to the law in (2) and the constraint of (5), the velocity at different frequencies and amplitudes are measured in detail. The results are shown in Fig.7. The maximal velocity is $\mathrm{v}=7.63 \mathrm{~cm} / \mathrm{s}$.

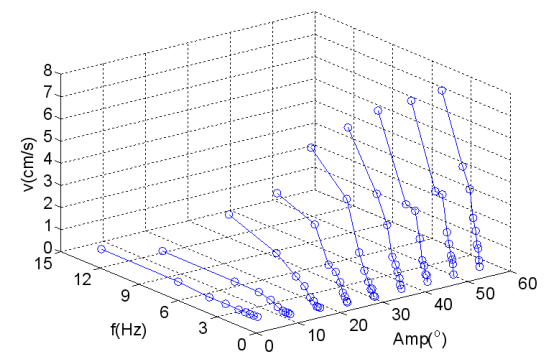

Fig. 7. The velocity at different frequencies and amplitudes

Experiment 2: Dynamic light source tracking

Firstly, the motion function $\mathrm{F}\left({ }^{*}\right)$ is built as a look-up table. The results of (21) are calculated offline and stored in the MCU. A light source moves randomly in the pool, and the robotic fish is put away from the light source. The trajectories are shown in the following:

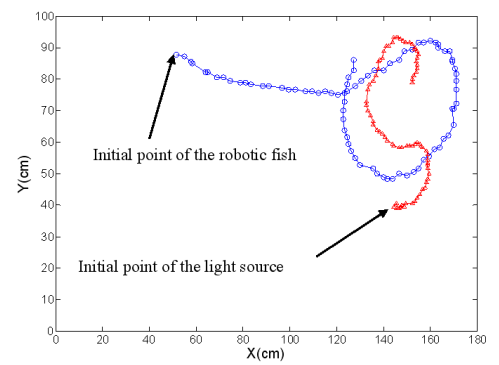

Fig. 8. The series of dynamic light source tracking 
where ' $\Delta$ ' is the trace of the light source, and ' $\mathrm{O}$ ' is the trace of robotic fish. $\mathrm{X}$ and $\mathrm{Y}$ in the figure are the edges of the pool which is regarded as obstacles in this experiment. From the figure, the robotic fish traces the light source without collision with the light source or pool edges.

\section{Conclusion}

In this paper, a miniature biomimetic robotic fish is given, which reduces the volume of the robotic fish largely. The method of propelling, turning, and Cturning for the robotic fish is proposed based on the motion law of Thunniform mode. The realtime path planning is given based on the sensors information. Experiments validate the design and algorithms.

\section{References}

1. M. S. Triantafyllou, Triantafyllou G S, An efficient swimming machine, Scientific American,vol.272(3), (1995), pp 64-70.

2. M. Sfakiotakis, D. M. Lane, and J. B. C. Davies, "Review of fish swimming modes for aquatic locomotion," IEEE J. Oceanic Eng. vol. 24, no. 2, (1999), pp. $237-252$

3. M. J. Lighthill, Note on the swimming of slender fish, J. Fluid Mech. 1960, vol.9: 305-317.

4. T. Y. Wu. Swimming of a waving plate, J. Fluid Mech. vol.10,1961, pp 321-344.

5. B. G. Tong, Propulsive mechanism of fishs undulatory motion, Mechanics In Engineering. vol. 22: 2000, pp 69-74.

6. N. Kato, Control Performance in the Horizontal Plane of a Robot fish with Mechanical Pectoral Fins, IEEE J. Ocean. Eng. vol. 25, 2000, pp.121-129.

7. T. Fukuda, A. Kawamoto, F. Arai, H. Matsuura, Mechnism and swimming experiment of micro mobile robot in water, Proceedings, IEEE Workshop on Micro Electro Mechanical Systems. 1994, pp 273-278.

8. J. Liu, H.S. Hu, Building a 3D simulator for autonomous navigation of robot fishes, IEEE IROS, 2004. pp 613C618

9. C. Zhou, Z. Cao, S. Wang and M. Tan, "The Posture Control and 3-D Locomotion Implementation of Biomimetic Robot Fish," IEEE IROS, pp. 2583$2588,(2006)$

10. W. Zhang, S. Guo and K. Asaka, "A New Type of Hybrid Fish-like Microrobot," IJAC. 4,2006 , pp. 358-365

11. J. Edd, S. Payen, B. Rubinsky and M. L. Stoller, "Biomimetic Propulsion for a Swimming Surgical Micro-Robot," IEEE IROS, (2003), pp. 2583-2588

12. Body/Caudal Fin Propulsion, Available at: http://www.ece.eps.hw.ac.uk/Research/oceans/projects/flaps/bcfmodes.htm

13. P. Domenfci, R.W. Blake "Kinematics and Performance of Fish Fast-start Swimming," J Exp BinI, (1997), 200, 1165-1178 